\title{
nature
}

\section{Vacuum at the heart of Europe}

A sudden withdrawal of support by the European Union for key scientific institutes could be a major setback for the continent's biological research and is yet another example of a lack of scientific vision at the European level.

S tick a pin anywhere on a map of Europe, and the country's head of state will at some point have sung fine words about how biology and biotechnology are top research priorities that, along with information technology, will fuel the industrial and medical revolutions of the next century. Such speeches inevitably include a warning that Europe needs to be "competitive with the United States and Japan" and that cooperation is one way of achieving this. Regrettably, a Scientifically United (or even Coherent) States of Europe is still a long way off, as illustrated by the disarray behind an impending crisis at the European Bioinformatics Institute (EBI) in Cambridge and other European-funded life-science centres in Europe (see pages 3 and 12).

EBI was launched as a joint venture in 1994 by the European Union (EU) and the European Molecular Biology Laboratory. But EU member states have now told the European Commission that it should no longer fund research infrastructure. Unless something happens soon, EBI, already a key facility for European molecular biologists, will wake up to the year 2000 minus half of its already pitiful budget of $\$ 8$ million - its US counterpart, the National Center for Biotechnology Information, will spend \$19 million this year.

Yet the dire consequences of this policy were not even discussed in the committees of representatives from the member states that devised it. If this Kafkaesque affair has any merit, it is that it has exposed the absence of a clear mechanism for the planning and support of research infrastructure at the European level. The European Commission has a clear mandate to coordinate what is better done at this rather than at the national level. But running large infrastructure on the short-term project grants the commission hands out is no substitute for long-term stable funding commitments.

In practice, such efforts have been driven forward largely through multilateral coordinated actions by the member states themselves. In the past, such deals have created pan-European institutions, such as the European Laboratory for Particle Physics (CERN), the European Synchrotron Radiation Facility and the science programme of the European Space Agency. These show that, when Europe gets its act together, it can excel.
But many of these programmes were launched by a generation of more visionary 'European' decision-makers. That vision currently seems to be lacking. The problem on infrastructure, as the EBI fiasco vividly illustrates, is that the member states have no generalized mechanism for making multilateral policy on scientific issues outside the commission. The patchy and ill-coordinated development of future European neutron and light sources illustrates the point. A coherent approach would have been for Europe to have first assessed its needs as a whole, and to have then worked out how best and most cost-effectively it could meet them collectively.

What is needed in Europe is not a new bureaucracy to deal with research infrastructure, but for science ministers and funding agencies to start thinking in terms of European solutions, and acting accordingly. At present, cooperation only takes place in earnest when the sums involved make this unavoidable, and even then it is often the lowest-common-denominator form of a project that prevails. Despite its enormous scientific importance, it is perhaps the relatively low costs of EBI which explain its low political profile so far.

The need for European cooperation is greatest in areas where the costs alone do not appear to make it essential. Most of Europe's research funds are scattered across national research councils and other funding agencies. Although local funding is often appropriate for investigator-driven research, the duplication of effort is frequently excessive, while many disciplines could benefit from greater concentration of much larger amounts of money.

But focusing substantial biomedical funds on strategic priorities inside a European National Institutes of Health, for example, is not for tomorrow. The EBI crisis should prompt the science agencies and their paymasters to complement their fine words about making European life sciences competitive on the world stage with vision, leadership and action. A decision by member states at the forthcoming meeting of the European Molecular Biology Laboratory to provide full funding for EBI as a short-term measure - and to register a firm commitment to negotiate a mechanism for the long-term support of this and similar initiatives — would be a good place to start.

\section{Fiction's futures}

A forward-looking series of articles depends at least as much on imagination as reality.

N othing dates as quickly as a prediction. That is why we have asked authors of our new weekly 'Futures' series, briefed to think about the scientific advances of the millennium to come, to concentrate on enjoying themselves rather than presenting anything especially serious or accurate. After all, when Nature was first published, 130 years ago today, predictions of the twenty-first century might have portrayed women in crinolines riding around in steam-driven airships.

This pursuit of happiness explains why we have asked, in the main, science-fiction writers to share their thoughts. As a genre, science fiction has purposes besides entertainment. It provides a medium in which writers can express not their predictions but their preoccupations with the present day. Moreover, such professional writers are perhaps better equipped than scientists to understand and convey how technological changes will affect the way we live, in all sorts of ways besides the mechanical. Nevertheless, we do expect scientists to contribute to this series, and we shall also be publishing other researchers' anticipations in a supplement at the beginning of next month.

In the weeks to come, the Futures series will present new writing from some of science fiction's household names - and some excellent new writing from authors of whom you might not have heard. For the first exercise in this new venture, Nature is delighted to publish an original contribution from Sir Arthur C. Clarke, perhaps science fiction's most famous name of all. You can find it on page 19. 